\title{
A Case Report of Canine Protothecosis
}

\author{
Satoshi HOSAKA ${ }^{1)}$ and Mari HOSAKA ${ }^{1)}$ \\ ${ }^{1)}$ Hosaka Animal Hospital, 4-17-1 Nihonmatsu, Sagamihara, Kanagawa 229-1137, Japan
}

(Received 22 April 2003/Accepted 16 January 2004)

ABSTRACT. A 10-year-old spayed mongrel dog was referred with repeated intercurrent hematochezia and anal bleeding. The dog was vigorous and had a normal appetite, and the fecal test showed no abnormal signs. Despite treatment primarily with sulfasalazine, the condition did not improve and unilateral blindness developed. A Prototheca zopfii infection was identified by further examination with bowel culture on Sabouraud's agar without cyclohexane and antibiotics. Subsequent to a vision loss in the other eye, the dog di ed showing signs of neurological disorder.

KEY WORDS: Prototheca zopfii.

Prototheca species are algae that lack chlorophyll, and reportedly cause a rare mycosis-like disease in a wide variety of mammals including humans, dogs, cats, cattle and pigs. Prototheca zopfii and Prototheca wickerhami are known to be pathogenic to dogs. Single infection of either species has been reported, except for a case caused by a mixed infection of $P$. zopfii and P. wickerhami [10]. Furthermore, Prototheca is ubiquitous in the environment and has been isolated from various sources such as tree sap, lake water, marine water and soil [4]. Although infections in cats and humans often occur as simple cutaneous lesions, systemic infection has been reported in canines [1-5, 8-10]. In this report, we present a systemic infection with $P$. zopfii in a canine. The case was resistant to symptomatic treatment and characterized by chronic bloody diarrhea followed by vision loss and neurological symptoms.

On October 25, 1998, a 10-year-old spayed mongrel dog was referred with a one-month history of hemafecia and anal bleeding. Although the dog had a normal appetite and was vigorous, it showed increased stool frequency, tenesmus, bleeding and loose or bloody mucous feces. The dog's bodyweight was $13.9 \mathrm{~kg}$, which was slightly lower than the reported weight of $15 \mathrm{~kg}$ prior to the onset of clinical signs, but the dog was not emaciated. The dog had no history of disease, and had been fed on meals prepared for humans. Fecal smear and flotation tests were both negative for parasite eggs and oocysts. No stenosis or tumor was detected by rectal palpation, but there was a blood clot on the inserted finger. A fecal parvovirus antigen test (PARVO TEST-LANIKKEN, Nikken Chemicals Co., Ltd., Tokyo, Japan) was also negative. Based on these results, we tentatively diagnosed ulcerative colitis, and treated the animal with sulfasalazine (10 mg/kg, BID). The symptoms improved transiently but not completely. After 16 days of treatment, the vision in the left eye was suddenly lost and retinal detachment was observed. As the dog had not responded to the medical treatment, we proceeded to further clinical examinations.

The complete blood count, blood chemistries and abdominal radiograph taken 25 days after referral were all within normal limits. A double contrast radiograph of the large intestine on the twenty-eighth day also showed no signs of abnormalities. Subsequently, the treatment was changed to metronidazole $(20 \mathrm{mg} / \mathrm{kg}$, BID). Biopsy was performed after 35 days of treatment for a histopathological examination. Samples were obtained from three parts of the descending colon and the associated swollen lymph nodes. As a potential cause of disease, a yeast-like organism, which was considered to be Prototheca, was observed in the mucosal layer of the colon and in the lymph nodes. The medical treatment was continued after the biopsy, and the feces became less loose and bloody, almost normal with a slight amount of bloody mucous. After 52 days of treatment, however, the vision of the right eye was also lost due to retinal detachment. Therefore, on the next day, feces and bowel samples were submitted to a testing center for human medicine (Showa Medical Science Corporation, Tokyo, Japan) for a definitive diagnosis. None of the fecal cultures on Sabouraud's agar containing cyclohexane, chloramphenicol (CP) or gentamycin (GM) detected Prototheca. Colonies of Prototheca, however, grew in the culture of the colon samples on Sabouraud's agar without cyclohexane or other antibiotics after 3 days, whereas the culture with cyclohexane, CP or GM did not show any sign of the organism. The organism in this culture was identified as $P$. zopfii from its carbohydrate assimilation pattern [6].

After 59 days of treatment, however, the dog was again referred with convulsions. At the time of referral, the dog was lying down hemorrhaging from the anus. A blood test indicated low blood glucose and leucopenia, and the dog died the following day. P. zopfii was found from a postmortem blood sample from the abdominal vena cava and the left vitreous humor material that were cultured on Sabouraud's agar without cyclohexane and other antibiotics (Showa Medical Science). The smear sample of vitreous humor showed protothecal masses of various sizes containing sporangiospores (Fig. 1). The fecal culture, on the other hand, showed no growth of the organism (Showa Medical Science).

The pathological examination (Hist. Vet. Inc, Kanagawa, 


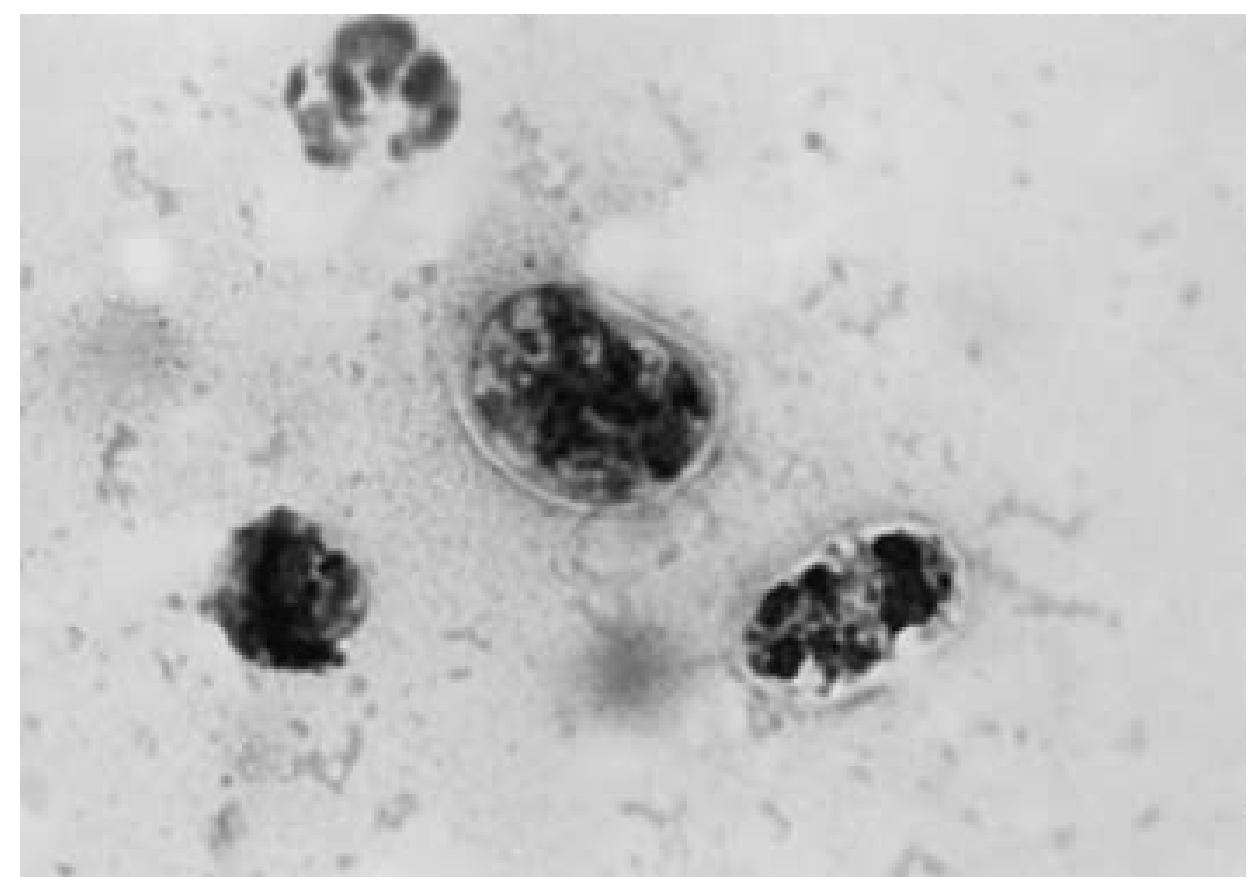

Fig. 1. Smear sample from the vitreous humor. Organisms of various sizes containing sporangiospores are observed. Giemsa stain; $\times 1,000$.

Japan) of the biopsy samples taken on the 35th day of treatment showed mild diffuse edema and mild-to-moderate cell infiltration in the large intestinal lamina propria mucosae. The cell infiltration was largely consisted of lymphocytes, plasma cells and a few disseminative large histiocytes. In addition, PAS-positive masses consisting of uneven ovalshaped or spore-like organisms were often found (Fig. 2). In the lymph node, similar inflammatory cells were observed along with PAS-positive organismal masses (Fig. 3). These findings indicated chronic diffuse colitis and hyperplasia in the lymph node.

Among the necropsy samples of the organs sent for histopathological screening, protothecal masses were found in the cardiac muscle and its interstitial spaces. The mass was formed by $20-40 \mu \mathrm{m}$, oval to spore-like organisms, and surrounding cardiac fibers showed mild degeneration accompanied by coagulative necrosis (Fig. 4). The other samples including heart, kidney, stomach and intestinal and mesenteric lymph nodes were also marked by multifocal nodular granulomatous inflammation caused by $P$. zopfii infection.

$P$. zopfii was identified by carbohydrate assimilation patterns, with a VITEK YBC Card (bioMerieux-Vitek Japan, Ltd, Tokyo, Japan) which was originally developed for identification of yeast-like fungi. In general, $P$. zopfii assimilates both glucose and glycerol, but only glucose on this card. The test may not differentiate $P$. zopfii from Blastoschizomyces capitatus or Candida krusei. Those three species can be subsequently identified by glucose fermentation test, true hyphae formation and conidial formation. $P$. zopfii is negative in all those tests and shows sporan- giospores by Gimsa stain. The yeast-like organism in our culture was determined to be $P$. zopfii based on those characteristics.

Antimicrobial susceptibility tests against $P$. zopfii (Showa Medical Science) was conducted on the 52nd day after the first referral for further treatment. The organism was found susceptible to amikacyn (AMK) and GM by the antibacterial test (Table 1), and also to amphotericin B and itraconazole by the antifungal test (Table 2). However, because the dog died before the results were obtained, these medicines were not administered to the dog. The minimum inhibitory concentration (MIC) values obtained can be used only as a reference, since neither of the test reagents was developed to test $P$. zopfii.

It has been suggested that suppressed immunity may largely account for the occurrence of canine protothecosis, considering the low incidence of the disease and the weak virulence of Prototheca [7, 8]. Breed-specific susceptibility has also been stated, since many of the cases have been reported in Collies $[4,9]$. In the present case, there was no underlying disease accounting for the cause of infection, and immunosuppression was not predicted. The dog may have had a genetic background that was susceptible to Prototheca such as in Collies. It is considered that Prototheca is taken in orally, then localizes in the colon and spreads throughout the body via the blood stream and the lymphatic system [9]. Although gastrointestinal symptoms are predominant in canine protothecosis, cutaneous, central nervous, lymphatic and ocular infections have also been observed [4]. The present case exhibited chronic colitis 


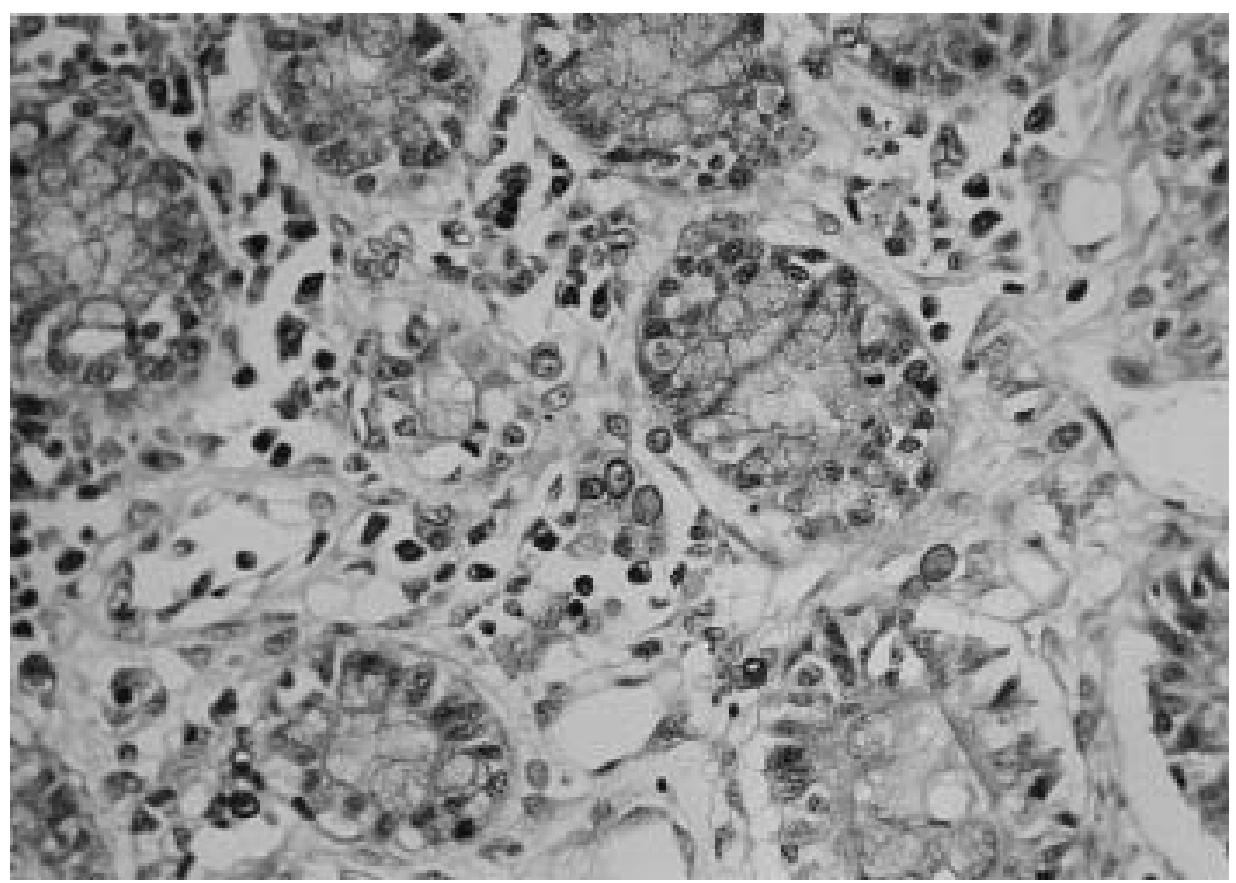

Fig. 2. Chronic inflammatory lesions in the colon showing diffuse infiltration of lymphocytes and plasma cells in the lamina propria mucosae. Protothecal organisms are observed as $20-40 \mu \mathrm{m}$, PAS positive and uneven oval to spore-like forms. PAS stain; $\times 200$.

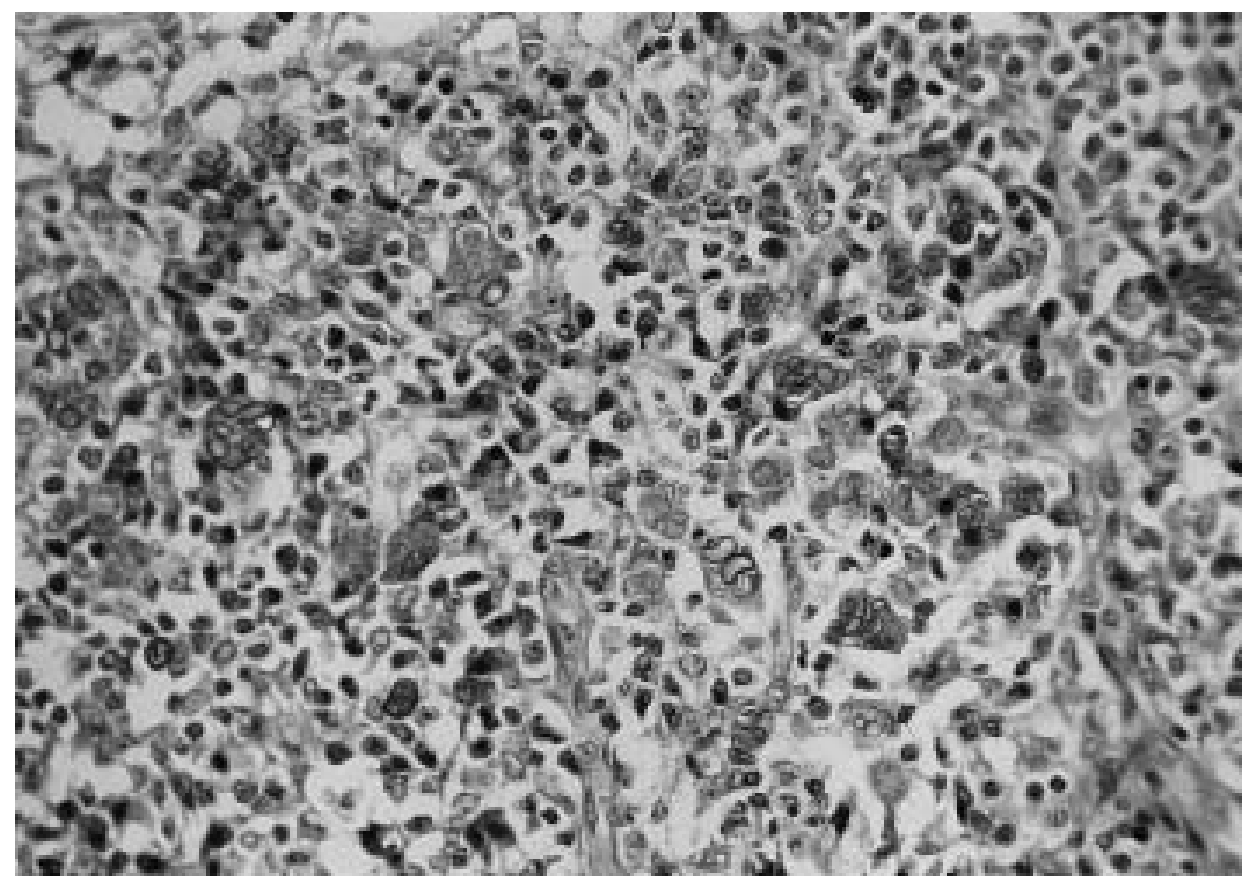

Fig. 3. Protothecal masses formed in medullary sinuses of the lymph node. $20-40 \mu \mathrm{m}$, PAS positive and uneven oval organisms are observed. PAS stain; $\times 200$.

with bleeding as a dominant symptom, then the lesion spread to other organs throughout the body. The observed chronic colitis is a common symptom in canine prototheco- sis [4], and the sudden blindness caused by retinal detachment, which started in the left eye, was also due to a $P$. zopfii infection as later confirmed by the vitreous culture. Pro- 


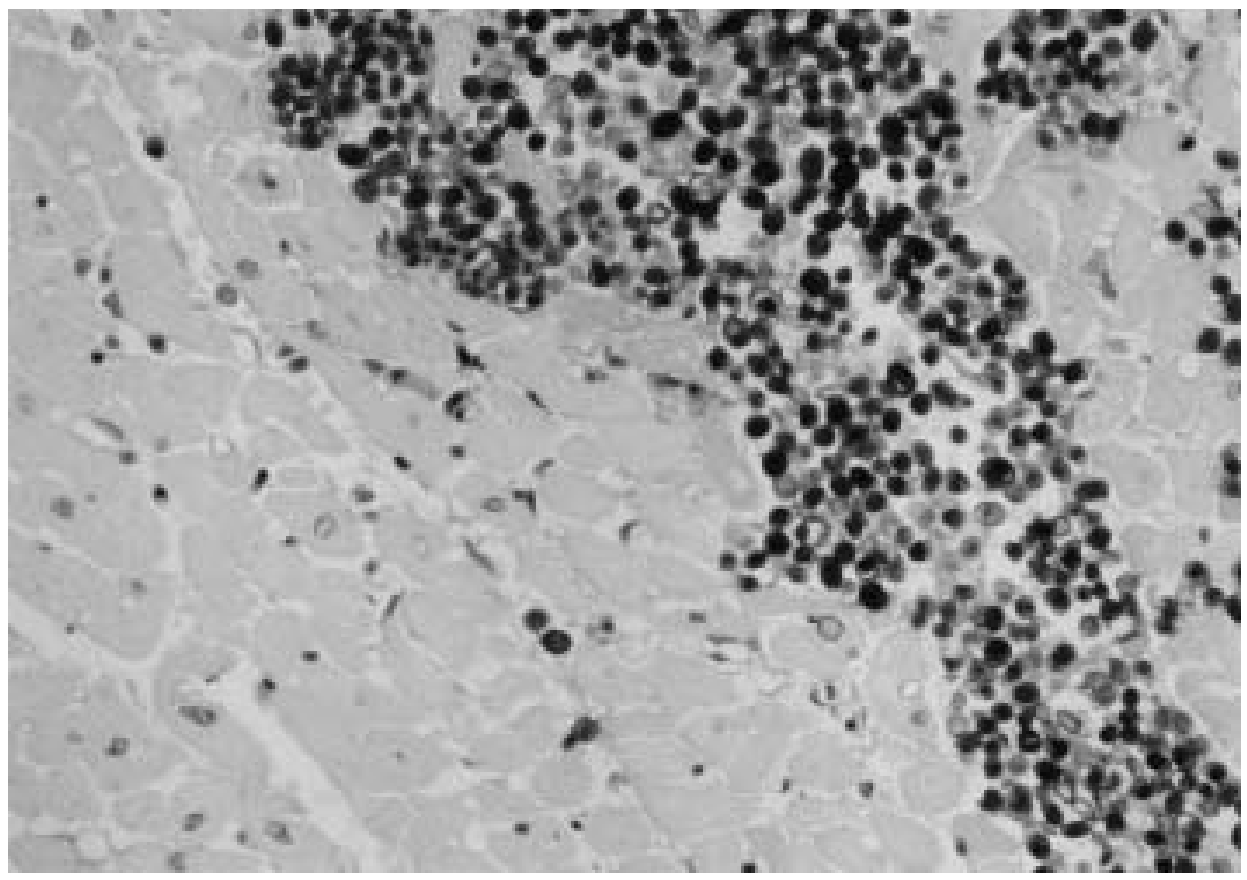

Fig. 4. A protothecal mass in the cardiac muscle formed by $20-40 \mu \mathrm{m}$, oval to spore-like organisms. Mild infiltration is recognized inside the mass. Surrounding cardiac muscle fibers are mildly degenerated. PAS stain; $\times 200$.

Table 1. Antibacterial susceptibility test

Reagent: Susceptibility test SHOWA DISC (Nissui Pharmaceuticals Co., Ltd, Tokyo, Japan)

\begin{tabular}{ll}
\hline Antibacterial agent & MIC $(\mu \mathrm{g} / \mathrm{m} l)$ \\
\hline Amikacyn & 3.13 \\
\hline Gentamycin & 1.56 \\
\hline Penicillin G & $\geq 25$ \\
\hline Aminobenzylpenicillin & $\geq 50$ \\
\hline Tetracycline & $\geq 25$ \\
\hline Chloramphenicol & $\geq 200$ \\
\hline Cehaplexin & $\geq 100$ \\
\hline Ofloxacin & $\geq 50$ \\
\hline Sulfamethoxazole-Trimethoprim & $\geq 800$ \\
\hline
\end{tabular}

totheca spp. do not grow on Sabouraud's agar cultured with cyclohexane or GM [3]. Consistent with this, the present case also did not show growth of Prototheca spp. on the culture with cyclohexane, GM and CP that were added in order to avoid microbial contamination. This suggests that the organism is susceptible to antimicrobials in vitro. Moreover, based on the results of the antimicrobial susceptibility tests, we have found that some of the antifungal agents as well as aminoglycoside antibiotics such as GM, inhibit Prototheca growth in vitro. However, antifungal agents lose
Table 2. Antifungal susceptibility test Reagent: ASTY (Antifungal Susceptibility Testing of Yeasts; Kyokuto Pharmaceuticals Co., Ltd, Tokyo, Japan)

\begin{tabular}{ll}
\hline Antifungal agent & MIC $(\mu \mathrm{g} / \mathrm{ml})$ \\
\hline Amphotericin B & 0.5 \\
\hline Fulcytosin & $\geq 64$ \\
\hline Fulconazole & $\geq 64$ \\
\hline
\end{tabular}

Reagent: Yeast-like Fungi FP (Eiken Chemicals Co., Ltd., Tokyo, Japan)

\begin{tabular}{ll}
\hline Antifungal agent & MIC $(\mu \mathrm{g} / \mathrm{m} l)$ \\
\hline Amphotericin B & 1 \\
\hline Itraconazole & 2 \\
\hline Fulcytosin & $\geq 64$ \\
\hline Fulconazole & $\geq 64$ \\
\hline Myconazole & $\geq 64$ \\
\hline
\end{tabular}

their effect on animals with protothecosis, and therefore a complete cure is difficult to attain [5]. This suggests that antifungal agents may have different susceptibility in vitro and in vivo. Considering the relationship between protothecal infection and host immunity, Prototheca may have been resistant to the drug in vivo in the present case. Also, Prototheca was not detected on any fecal cultures in this case, 
similarly to a previous report [9]. Therefore, early noninvasive diagnosis is impossible. Acute blindness may be the chief complaint or initial symptom that owners notice, as has been in other cases $[1,2]$.

Protothecal infection is often left unnoticed in the early stages, thus reducing the chances for appropriate treatment. Therefore, by the time the organism is detected, the disease may have become resistant to symptomatic treatment. When hemorrhagic diarrhea is accompanied with blindness, early diagnosis by biopsy should be sought. If immune stimulants or antifungal drugs are applied in the early stages, they might be a useful therapy for protothecosis.

\section{REFERENCES}

1. Blogg, J. R. and Sykes, J. E. 1995. Aust. Vet. J. 72: 147-149.
2. Cook, J. R. Jr., Tyler, D. E., Coulter, D. B. and Chandler, F. W. 1984. J. Am. Vet. Med. Assoc. 184: 1266-1271.

3. Gaunt, S. D., McGrath, R. K. and Cox, H. U. 1984. J. Am. Vet. Med. Assoc. 185: 906-907.

4. Migaki, G., Font, R. L., Sauer, R.M., Kaplan, W. and Miller, R. L. 1982. J. Am. Vet. Med. Assoc. 181: 794-797.

5. Moore, F. M., Schmidt, G. M., Desai, D. and Chandler, F. W. 1985. J. Am. Vet. Med. Assoc. 186: 705-708.

6. Padhye, A.A., Baker, J.G. and D'amato, R.F. 1979. J. Clin. Microbiol. 10: 579-582.

7. Perez, J., Ginel, P. J., Lucena, R., Hervas, J. and Mozos, E. 1997. J. Comp. Pathol. 117: 83-89.

8. Rakich, P. M. and Latimer, K. S. 1984. J. Am. Vet. Med. Assoc. 185: 681-683.

9. Thomas, J. B. and Preston, N. 1990. Aust. Vet. J. 67: 25-27.

10. Tyler, D. E., Lorenz, M. D., Blue, J. L., Munnell, J. F. and Chandler, F. W. 1980. J. Am. Vet. Med. Assoc. 176: 987-993. 